\title{
The role of lymphadenectomy in high risk prostate cancer
}

\author{
Fiona C. Burkhard · Urs E. Studer
}

Received: 22 February 2008 / Accepted: 26 February 2008 / Published online: 28 March 2008

(C) Springer-Verlag 2008

\begin{abstract}
Historically, patients with high risk prostate cancer were considered poor candidates for radical prostatectomy (RP) due to the likelihood of positive pelvic lymph nodes and decreased long term survival. Although there is still no consensus on the optimal therapy for this group of patients, there is increasing evidence that surgery could play a role. Cancer specific survival (CSS) rates after RP for locally advanced disease at 10 year follow up range from 29 to $72 \%$, depending on tumor differentiation. The role of pelvic lymph node dissection (PLND) in prostate cancer remains a controversial topic. Nonetheless, in conjunction with RRP extended PLND (ePLND) should be performed as extended lymph node dissection in lieu of standard PLND may increase staging accuracy, influence decision making with respect to adjuvant therapy and possibly impact outcome. High risk patients with organ confined prostate cancer and low volume (micro)metastatic disease may be the ones to profit most from this approach.
\end{abstract}

Keywords High risk prostate cancer - Lymphadenectomy . Nodal metastases $\cdot$ Radical prostatectomy

\section{Introduction}

Historically, patients with high risk prostate cancer were considered poor candidates for radical prostatectomy (RP) due to the likelihood of positive pelvic lymph nodes and decreased long term survival $[1,2]$. Although there is still no consensus on the optimal therapy for this group of

F. C. Burkhard $(\square) \cdot$ U. E. Studer

Department of Urology, University of Bern,

Inselspital, Bern, Switzerland

e-mail: fiona.burkhard@insel.ch patients, there is increasing evidence that surgery could play a role. The initiation of PSA screening, has led to stage migration and improved outome after RP rekindling the interest in surgery as an option for patients with high risk prostate cancer. In locally advanced disease (cT3) the cancer specific survival (CSS) rate after RP at 10 year follow up is $57-72 \%$ [1, 3, 4]. Tumor differentiation is an important confounding factor and in well differentiated and moderately differentiated cT3 tumors CSS rates at 10 years range from $67-85 \%$, however in poorly differentiated tumors CSS rates sink to approx. 29\% [1, 4].

The role of pelvic lymph node dissection (PLND) in prostate cancer remains a controversial topic. There is no doubt that an adequate PLND improves staging and as a consequence allows better assessment of the disease and it's prognosis. It's benefical effect on disease progression and survival, however can be questioned, especially in patients with low risk prostate cancer. Patients with high risk prostate cancer may be the ones most likely to profit from ePLND, especially those with micrometastatic disease. Patients with more extensive lymph node metastases may benefit from androgen deprivation therapy (ADT) [5].

\section{Definition of high risk prostate cancer}

The definition of high risk prostate cancer varies throughout the literature. Where some consider a PSA $>20 \mathrm{ng} / \mathrm{ml}$ or a Gleason sum $>7$ or primary Gleason pattern of four or five or clinical stage T3a to be high risk, others consider a PSA $\geq 15 \mathrm{ng} / \mathrm{ml}, \geq \mathrm{cT} 3$ or biopsy Gleason score $>7$ high risk prostate cancer $[6,7]$. The AUA clinical guidelines define high risk as a PSA $>20 \mathrm{ng} / \mathrm{mL}$ or a Gleason score of $8-10$ or clinical stage $\geq \mathrm{T} 2 \mathrm{c}$ [8]. Using eight different definitions of high risk prostate cancer Yossepowitch et al. 
[9] assessed a study population of 4,708 men treated by RRP and concluded that: "patients diagnosed with high risk prostate cancer by currently available definitions do not have a uniformly poor prognosis after radical prostatectomy". Of the high risk tumors $22-63 \%$ proved to be confined to the prostate, 7-23\% had lymph node metastasis and the 5 year relapse free probability after RP was $49 \%$. The conclusion was that in high risk prostate cancer, RP monotherapy may be an acceptable treatment option and that many cancers in this group are confined to the prostate.

PSA values are not specific for prostate cancer, however PSA dynamics may be. Both a short PSA doubling time and an increased PSA velocity have been associated with poor prognosis, however clear cut definitions are still lacking $[10,11]$. Although PSA dynamics may well prove to be one of the most important factors to help predict patients at risk of death from prostate cancer, they are not likely to reflect the actual course of disease in all patients. PSA changes may only provide usefull information in a few patients at a time when the disease is still localized and the patient will truly profit from treatment. Indeed many patients without an accelerated PSA doubling time or velocity at the time of local therapy with curative intent will later progress [12]. In addition PSA negative tumors may have misleading PSA values. To summarize, the definition of high risk prostate cancer has yet to be standardized and future efforts need to be focused on developing methods to discriminate between patients at high risk for relapse who may benefit from local therapy alone and those requiring an aggressive combination therapy.

\section{Clinical staging}

Accurate staging defines the extent and location of disease, helps predict malignant potential, influences decision making with respect to therapeutic options and possibly has an impact on outcome. Efforts are being made to improve preoperative diagnosis of lymph node metastasis and so minimize surgical risks and costs. Predictive nomograms that help estimate the likelihood of positive nodes for each individual patient by assigning points for specific risk factors have been developed. However, most of these nomograms are based on limited PLND and therefore their applicability is questionable. They may be adequate to estimate the incidence of positive nodes in a limited field of resection, they do not however reflect the true probability of lymph node metastases.

Recently, Briganti et al. found that the risk of positive nodes in men undergoing RRP increases linearly in proportion to the number of lymph nodes removed, hereby confirming previous observations by other authors [13, 14]. They then proceeded to develop and validate a nomogram that predicts the probability of lymph node invasion based on clinical parameters (PSA level, clinical stage, biopsy Gleason score sum) and the number of nodes removed during PLND [15]. The aim of this nomogram is to estimate the optimal number of nodes that should be removed. As with all other nomograms its accuracy depends on the cohort used for it's development. For one the majority of patients included qualify as low risk patients and 9\% (total 71 patients) were lymph node positive, this rather small number limiting the significance. In addition, true ePLND was performed only in $23 \%$ of patients and this combined with the knowledge that primary lymphatic drainage sites may go up to the inferior mesenteric artery which was not accounted for in the nomogram may again limit it's reliability [16]. In knowledge of the interindividual variability of lymph node distribution, it appears easier to either perform an ePLND including the known areas of prostatic drainage or to omit PLND. Furthermore, the aim of PLND should not only be to determine if a patient has positive nodes, but also to remove as many nodes as possible. Nomograms can only attempt to predict the probability of finding positive nodes in a large series of patients and their value for the individual patient remains questionable. The use of CT scans or MRI to evaluate lymph node involvement is not routinenly recommended as the low sensitivity of $10-30 \%$ for imaging pelvic lymph node metastases is not acceptable in high risk patients [17].

The sentinel lymph node (SLN) concept has been applied to various malignant tumors. The SLN a term introduced by Cabanas [18] is the node where lymphatic flow from the tumor first arrives. Wawroschek et al. [19] introduced the radioisotope method for detection of SLN in prostate cancer. In their study the SLN method and the consecutive lymphadenectomy showed an apparently high sensitivity rate of $96 \%$. However the study had certain weaknesses. The gamma probe needs to be in very close contact with the tissue to get a good signal and lymph nodes outside the area explored or with a weak signal may be missed. Since it is known that a significant number of positive nodes are also found along the internal iliac vessels, the common iliac vessels, in the presacral and paraaortic tissue, areas which were not all explored, some could have been missed with the possibility of incomplete sampling [16, 20, 21]. Takashima et al. [22] using the SLN technique were only able to detect in vivo $40 \%$ of the hot nodes detected ex vivo after extended PLND. Anatomic localization of prostatic sentinel nodes with fusion imaging of single photon emission computed tomography (SPECT) and CT scans after intraprostatic injection of Technetium-99m-Nanocolloid may be a more reliable imaging technique but is time consuming, costly and is dependent on the skills of the nuclear medicine specialist [23]. In addition in significant nodal disease technetium uptake can be compromised so 
over $1 / 3$ of positive nodes remain unidentified [24]. Other new and potentially promising techniques such as high resolution MRI with lymphotrophic superpara-magentic nanoparticles are being developed and evaluated, however detection of micrometastasis remains limited and these techniques are as yet not available [25]. The inability to accurately diagnose minor lymph node metastasis preoperatively and the important prognostic information gained by identifying nodal disease emphasizes the role of extended PLND during radical retropubic prostatectomy (RRP).

\section{Where to look for the nodes in high risk prostate cancer}

A standardized universal definition of limited/ extended PLND for prostate cancer does not exist. The minimal template only includes lymphatic tissue from the obturator fossa, the standard variant also includes lymphatic tissue along external iliac vein. Extended PLND additionally includes lymphatic tissue along the internal iliac vessels both medially and laterally and in some cases along the common iliac vessels. The next question is where to look for the positive nodes. Evidence suggests that increased lymph node sampling outside the obturator fossa may increase the detection of metastatic disease. Heidenreich et al. [26] reported on 103 patients who had an extended PLND comprising the external iliac, internal iliac, obturator, and common iliac lymph nodes bilaterally and the presacral nodes. They compared this group of patients with 100 men who received only standard PLND. Lymph node metastases were diagnosed in $26 \%$ in the extended group and in only $12 \%$ in the standard PLND group. Despite negative obturator $\mathrm{LN}$, positive $\mathrm{LN}$ were identified in the internal iliac and presacral regions. Forty-two percent of all LN metastases were detected outside the regions of standard PLND. Wawroschek et al. by extending the region of lymphadenectomy to include not only the tissue in the external iliac region and fossa obturatoria but also the tissue along the internal iliac vessels, in the paravesical, presacral and pararectal tissue they detected an additional 35\% of lymph node positive patients [27]. Our group reported the results following meticulous PLND along the external iliac vein, the obturator nerve, and the internal iliac (hypogastric) vessels in men with clinically organ-confined disease. Eightyeight of 365 men (24\%) had positive LN. Internal iliac LN were positive in $58 \%$ and internal iliac $\mathrm{LN}$ alone were positive in $19 \%$ of the men [20]. Similar results have been reported using the laparoscopic approach [28]. Stone et al. [28] reported twice as many $\mathrm{LN}$ removed via extended than limited laparoscopic PLND (mean 17:8 vs. 9.3), and three times as many patients with $\mathrm{LN}$ metastasis in the extended group [23.1 vs. $7.3 \% \quad(p=0.02)]$. However, the link between the extent and the yield of PLND was not always confirmed. The only randomized study on extended versus limited PLND was reported by Clark et al. [29], who found no difference in the yield of positive nodes with more extended dissection (extended: $3.2 \%$ vs. limited: $2.4 \%$ ). The two types of dissection were conducted in the same patient on contralateral sides, however, the majority of patients had a low probability of LN metastases and therefore would not have required a PLND. Comparing these two techniques in a cohort who are unlikely to have positive LN obviously limits the power of the trial. Even without this limitation, the number of patients examined was inadequate for an equivalence study. In addition, $90 \%$ of their patients had T1c or T2a disease, which is mostly a unilateral disease, and therefore randomly assigning them to extended PLND on only one side carries a substantial risk that the extended PLND was performed on the non tumor bearing side. Also, neither the number of nodes removed, nor the histopathological work-up were defined. Therefore, the study design renders it hard to compare their data with those of other previous studies or draw any conlusions for high risk patients.

A consensus on terminology of PLND is desperately needed. In addition as a result of new insights the optimal extent of PLND is continually being redefined. Recent data using intraprostatic technetium injections and fused singlephoton emission tomography (SPECT) combined with either CT or MRI to map lymphatic drainage from the prostate showed that the prostate has multiple landing sites and that the extended template may need modification as potentially $30-40 \%$ of the lymphatic landing sites are not included if PLND is limited to the iliac vessels distal the bifurcation of the common iliac artery [16]. By extending the dissection along common iliac vessels at least up to the ureteric crossing approximately $75 \%$ of all nodes potentially harbouring metastasis can be removed (Fig. 1).

Taken together, the above data shows that, in general, extended PLND yields higher rates of positive LN. Importantly, several studies demonstrated that up to two thirds of all patients with $\mathrm{LN}$ metastasis have positive nodes along the internal iliac vessels, an area not included in a limited PLND. A PLND, should therefore always include at least the internal iliac tissue on both sides, so identifying $\mathrm{LN}$ invasion that would remain undetected following limited PLND. By extending the template to include the tissue along the common iliac vessels up to the ureteric crossing $75 \%$ of all nodes potentially harbouring metastases are removed.

\section{Therapeutic consequences of extended PLND}

While a therapeutic benefit of PLND has been questined by some authors, the possibility of a therapeutic benefit for PLND in prostate cancer has been suggested by others. 

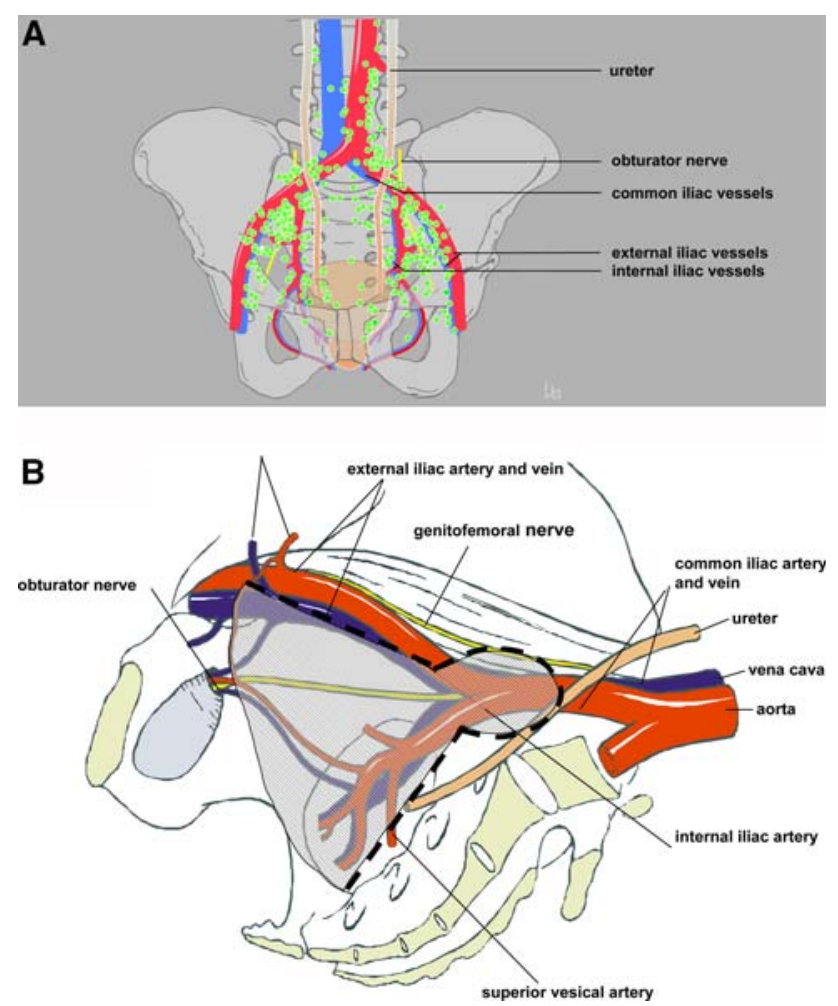

Fig. 1 a Primary landing sites of the prostate determined by intraprostatic technetium injections and fused single-photon emission tomography (SPECT) combined with either CT or MRI. b Proposed template including tissue along the common iliac vessels up to the crossing of the ureter

DiMarco et al. [30] did not observe an inter-relation between the extent of PLND and prostate cancer outcome in $\mathrm{LN}$ negative men, nor did they find a survival difference when comparing the results of extended PLND (14 nodes removed) between 1987 and 1989 with those of a more limited PLND (5 nodes removed) from 1999 to 2000. However, possible $\mathrm{T}$-stage migration, with higher tumor stages in the earlier period, was not taken into account. Because an extended PLND in the earlier period led to similar results for disease progression and survival as removing fewer nodes in the later period, their results may ultimately imply that the extended PLND had a therapeutic role for the earlier cohort. Recently, Masterson et al. [31] examined the association between the number of LNs removed, the number of positive LNs and disease progression in patients with clinically localized prostate cancer. They found that in men without nodal involvement an increased number of nodes removed correlated significantly with freedom from biochemical recurrence. Similarly, Joslyn and colleagues, using the SEER database, concluded that patients with LN involvement had a significantly greater number of nodes removed compared with those with no LN involvement and that extended PLND reduces the long-term risk of prostate cancer related death, even in patients with negative nodes compared to patients without PLND [14]. Recently Bader et al. [13] demonstrated that the rate of tumor progression is higher in pNO patients with few nodes removed than in patients with a larger number of negative nodes removed. A potential explanation for these observations is that a thorough nodal resection eliminated micrometastases that were not detected by routine histologic examination. This hypothesis is reported by recent studies applying special assays to determine occult metastasis in pathologically lymph node negative patients [32-34]. These investigators found occult metastasis in up to $30 \%$ of patients and concluded that a significant benefit in biochemical recurrencefree survival might exist for patients with micrometastases in a few nodes undergoing an extended dissection. It seems therefore that not only detection of diseased nodes but removal of as many primary lymphatic landing sites as possible should be the main objective for PLND to improve outcome.

This is supported by further evidence indicating that patients with minimal LN metastases will have better prognosis by removing the diseased nodes. Golimbu and coworkers [35] retrospectively analyzed 42 patients with nodal disease who underwent PLND and RRP. In this series, patients with low tumor bulk and one positive LN had survival rates comparable to those of matched controls after a mean follow-up of 5 years. Catalona suggested that treatment may be curative even in LN positive disease, however the 6 year follow up may be too short to allow a definite statement. In a small series of 12 patients with LN involvement and no adjuvant therapy, $75 \%$ remained recurrence free at 5 years and $58 \%$ at 7 years [36]. The results reported by Pound et al. [37] for patients with LN micrometastasis, revealed a 10-year metastasis-free survival rate of $68 \%$, again without adjuvant therapy. In 2003, Bader et al. reported on 92 men followed with histologically proven LN metastases who received no adjuvant therapy. After 45 months (median), 15 of 39 patients with only one positive node remained without signs of progression [38]. Recently Boorjian and colleagues [39] retrospectively reviewed a large series of patients with positive lymph nodes after RRP and PLND, although with a limited number of nodes (median 11) removed. The majority of the node positive patients received androgen deprivation therapy. In their series $56 \%$ of the patients had a 10 year BPFS. In Pagliarulos group of initially node negative high risk patients with immunohistochemically detected occult lymph node metastasis there was an estimated 39\% probability of remaining biochemically disease free for 10 years [34]. This did not differ significantly from the group of patients who were pathologically lymph node positive. Interestingly after a median observation time of 12.9 years only half of the patients with PSA relapse also developed clinical recurrence. 
Prognosis has also been shown to depend on the number of positive nodes involved. Daneshmand et al. [40] reported that patients who had one or two positive LNs had a clinical recurrence-free survival rate of 70 and $73 \%$ at 10 years. In our series 10 and $14 \%$ of patients with two or more positive LN remained disease free [38]. By contrast, men with five or more involved nodes had a recurrence-free survival rate of only $49 \%$. Boorjian found that one lymph node metastasis increased the risk of cancer specific death almost 4-fold, which in patients with two or greater positive lymph nodes was increased 2-fold compared to that in patients with one positive node [39].

Opponents of extended PLND argue that performing an extended PLND results in increased morbidity and costs [41]. Morbidity can be minimized if attention is paid to a few details: (1) Ligation of lymphatic vessels coming from the legs, instead of clipping. Hemoclips have a tendency to be torn away during subsequent surgery. (2) Placement of two drains one on each side of the pelvis where PLND was performed. Drains should be removed gradually until the total amount drained is less than $50 \mathrm{ml} / 24 \mathrm{~h}$. (3) Injection of low molecular heparin into the upper arm instead of the thigh.

\section{Conclusions}

There is evidence suggesting that RRP monotherapy is a treatment option in selected high risk patients. In conjunction with RRP extended PLND should be performed as extended lymph node dissection in lieu of limited PLND may increase staging accuracy, influence decision making with respect to adjuvant therapy and possibly impact outcome. High risk patients with localized prostate cancer and low volume (micro)metastatic disease may be the ones to profit most from this approach.

\section{References}

1. Gerber GS, Thisted RA, Chodak GW, Schroder FH, Frohmuller HG, Scardino PT, Paulson DF, Middleton AW Jr, Rukstalis DB, Smith JA Jr et al (1997) Results of radical prostatectomy in men with locally advanced prostate cancer: multi-institutional pooled analysis. Eur Urol 32:385-390

2. Khan MA, Mangold LA, Epstein JI, Boitnott JK, Walsh PC, Partin AW (2004) Impact of surgical delay on long-term cancer control for clinically localized prostate cancer. J Urol 172:18351839

3. Lerner SE, Blute ML, Zincke H (1995) Extended experience with radical prostatectomy for clinical stage $\mathrm{T} 3$ prostate cancer: outcome and contemporary morbidity. J Urol 154:1447-1452

4. van den Ouden D, Hop WC, Schroder FH (1998) Progression in and survival of patients with locally advanced prostate cancer (T3) treated with radical prostatectomy as monotherapy. J Urol 160:1392-1397
5. Messing E, Manola J, Sarosdy M, Wilding G, Crawford D, Trump D (2003) Immediate hormonal therapy compared with observation after radical prostatectomy and pelvic lymphadenectomy in men with node positive prostate cancer; results at 10 years of EST 3886. J Urol 169:396

6. D’Amico AV, Schultz D, Loffredo M, Dugal R, Hurwitz M, Kaplan I, Beard CJ, Renshaw AA, Kantoff PW (2000) Biochemical outcome following external beam radiation therapy with or without androgen suppression therapy for clinically localized prostate cancer. Jama 284:1280-1283

7. Scardino P (2005) Update: NCCN prostate cancer Clinical Practice Guidelines. J Natl Compr Canc Netw 3(Suppl 1):S29-S33

8. Thompson I, Thrasher JB, Aus G, Burnett AL, Canby-Hagino ED, Cookson MS, D'Amico AV, Dmochowski RR, Eton DT, Forman JD et al (2007) Guideline for the management of clinically localized prostate cancer: 2007 update. J Urol 177:2106-2131

9. Yossepowitch O, Eggener SE, Bianco FJ Jr, Carver BS, Serio A, Scardino PT, Eastham JA (2007) Radical prostatectomy for clinically localized, high risk prostate cancer: critical analysis of risk assessment methods. J Urol 178:493-499 (discussion 499)

10. D'Amico AV, Chen MH, Catalona WJ, Sun L, Roehl KA, Moul JW (2007) Prostate cancer-specific mortality after radical prostatectomy or external beam radiation therapy in men with 1 or more high-risk factors. Cancer 110:56-61

11. Carter HB, Ferrucci L, Kettermann A, Landis P, Wright EJ, Epstein JI, Trock BJ, Metter EJ (2006) Detection of life-threatening prostate cancer with prostate-specific antigen velocity during a window of curability. J Natl Cancer Inst 98:1521-1527

12. Carter HB, Kettermann A, Ferrucci L, Landis P, Metter EJ (2007) Prostate-specific antigen velocity risk count assessment: a new concept for detection of life-threatening prostate cancer during window of curability. Urology 70:685-690

13. Bader P, Spahn M, Huber R, Echtle D, Frohneberg D (2004) Limited lymph node dissection in prostate cancer may miss lymph node metastasis and determines outcome of apparently pN0 prostate cancer. Eur Urol 3:16 (A 55)

14. Joslyn SA, Konety BR (2006) Impact of extent of lymphadenectomy on survival after radical prostatectomy for prostate cancer. Urology 68:121-125

15. Briganti A, Chun FK, Salonia A, Gallina A, Farina E, Da Pozzo LF, Rigatti P, Montorsi F, Karakiewicz PI (2006) Validation of a nomogram predicting the probability of lymph node invasion based on the extent of pelvic lymphadenectomy in patients with clinically localized prostate cancer. BJU Int 98:788-793

16. Mattei A, Fuechsel FG, Dhar NB, Warncke SH, Thalmann GN, Krause T, Studer UE (2007) The template of the primary lymphatic landing sites of the prostate should be revisited: results of a multimodality mapping study. Eur Urol 53:118-125

17. Borley N, Fabrin K, Sriprasad S, Mondaini N, Thompson P, Muir G, Poulsen J (2003) Laparoscopic pelvic lymph node dissection allows significantly more accurate staging in "high-risk" prostate cancer compared to MRI or CT. Scand J Urol Nephrol 37:382-386

18. Cabanas RM (1977) An approach for the treatment of penile carcinoma. Cancer 39:456-466

19. Wawroschek F, Vogt H, Weckermann D, Wagner T, Hamm M, Harzmann R (2001) Radioisotope guided pelvic lymph node dissection for prostate cancer. J Urol 166:1715-1719

20. Bader P, Burkhard FC, Markwalder R, Studer UE (2002) Is a limited lymph node dissection an adequate staging procedure for prostate cancer? J Urol 168:514-518 (discussion 518)

21. Heidenreich A, Varga Z, Olbert P, Hofmann R (2001) Radical pelvic lymphadenectomy in clinically localised prostate cancer: high frequency of atypical metastasis. Eur Urol 39(suppl. 5):135 (A 530)

22. Takashima H, Egawa M, Imao T, Fukuda M, Yokoyama K, Namiki M (2004) Validity of sentinel lymph node concept for patients with prostate cancer. J Urol 171:2268-2271 
23. Mattei A, Fuechsel FG, Warncke S, Z'Brun S, Baermann C, Krause TM, Studer UE (2005) Advantages of preoperative imaging with Technetium-99m-nanocolloid for prostate-sentinel-lymphnode-adenectomy: accuracy and surgical detection time using presurgical imaging guidance prior to gamma-probe-detection versus gamma-probe detection alone. J Urol 173:433

24. Gronau E, Weckermann D, Harzmann R (2005) Sentinel lymph node resection in prostate cancer patients with a PSA higher than $20 \mathrm{ng} / \mathrm{ml}$. J Urol 173:437

25. Harisinghani MG, Barentsz J, Hahn PF, Deserno WM, Tabatabaei S, van de Kaa CH, de la Rosette J, Weissleder R (2003) Noninvasive detection of clinically occult lymph-node metastases in prostate cancer. N Engl J Med 348:2491-2499

26. Heidenreich A, Varga Z, Von Knobloch R (2002) Extended pelvic lymphadenectomy in patients undergoing radical prostatectomy: high incidence of lymph node metastasis. J Urol 167:1681-1686

27. Wawroschek F, Wagner T, Hamm M, Weckermann D, Vogt H, Markl B, Gordijn R, Harzmann R (2003) The influence of serial sections, immunohistochemistry, and extension of pelvic lymph node dissection on the lymph node status in clinically localized prostate cancer. Eur Urol 43:132-137

28. Stone NN, Stock RG, Unger P (1997) Laparoscopic pelvic lymph node dissection for prostate cancer: comparison of the extended and modified techniques. J Urol 158:1891-1894

29. Clark T, Parekh DJ, Cookson MS, Chang SS, Smith ER Jr, Wells N, Smith JA Jr (2003) Randomized prospective evaluation of extended versus limited lymph node dissection in patients with clinically localized prostate cancer. J Urol 169:145-147 (discussion 147-148)

30. DiMarco DS, Zincke H, Sebo TJ, Slezak J, Bergstralh EJ, Blute ML (2005) The extent of lymphadenectomy for pTXNO prostate cancer does not affect prostate cancer outcome in the prostate specific antigen era. J Urol 173:1121-1125

31. Masterson TA, Bianco FJ Jr, Vickers AJ, DiBlasio CJ, Fearn PA, Rabbani F, Eastham JA, Scardino PT (2006) The association between total and positive lymph node counts, and disease progression in clinically localized prostate cancer. J Urol 175:1320-1324 (discussion 1324-1325)
32. Ferrari AC, Stone NN, Kurek R, Mulligan E, McGregor R, Stock R, Unger P, Tunn U, Kaisary A, Droller M et al (2006) Molecular load of pathologically occult metastases in pelvic lymph nodes is an independent prognostic marker of biochemical failure after localized prostate cancer treatment. J Clin Oncol 24:3081-3088

33. Miyake H, Hara I, Kurahashi T, Inoue TA, Eto H, Fujisawa M (2007) Quantitative detection of micrometastases in pelvic lymph nodes in patients with clinically localized prostate cancer by realtime reverse transcriptase-PCR. Clin Cancer Res 13:1192-1197

34. Pagliarulo V, Hawes D, Brands FH, Groshen S, Cai J, Stein JP, Lieskovsky G, Skinner DG, Cote RJ (2006) Detection of occult lymph node metastases in locally advanced node-negative prostate cancer. J Clin Oncol 24:2735-2742

35. Golimbu M, Morales P, Al-Askari S, Brown J (1979) Extended pelvic lymphadenectomy for prostatic cancer. J Urol 121:617-620

36. Catalona WJ, Miller DR, Kavoussi LR (1988) Intermediate-term survival results in clinically understaged prostate cancer patients following radical prostatectomy. J Urol 140:540-543

37. Pound CR, Partin AW, Eisenberger MA, Chan DW, Pearson JD, Walsh PC (1999) Natural history of progression after PSA elevation following radical prostatectomy. Jama 281:1591-1597

38. Bader P, Burkhard FC, Markwalder R, Studer UE (2003) Disease progression and survival of patients with positive lymph nodes after radical prostatectomy. Is there a chance of cure? J Urol 169:849-854

39. Boorjian SA, Thompson RH, Siddiqui S, Bagniewski S, Bergstralh EJ, Karnes RJ, Frank I, Blute ML (2007) Long-term outcome after radical prostatectomy for patients with lymph node positive prostate cancer in the prostate specific antigen era. J Urol 178:864870 (discussion 870-861)

40. Daneshmand S, Quek ML, Stein JP, Lieskovsky G, Cai J, Pinski J, Skinner EC, Skinner DG (2004) Prognosis of patients with lymph node positive prostate cancer following radical prostatectomy: long-term results. J Urol 172:2252-2255

41. Campbell SC, Klein EA, Levin HS, Piedmonte MR (1995) Open pelvic lymph node dissection for prostate cancer: a reassessment. Urology 46:352-355 